Voix et Images

voixetimages

\title{
Une autre lecture de Nelligan
}

\section{Roger Chamberland}

Volume 18, numéro 2 (53), hiver 1993

Francine Noël

URI : https://id.erudit.org/iderudit/201030ar

DOI : https://doi.org/10.7202/201030ar

Aller au sommaire du numéro

\section{Éditeur(s)}

Université du Québec à Montréal

\section{ISSN}

0318-9201 (imprimé)

1705-933X (numérique)

Découvrir la revue

\section{Citer cet article}

Chamberland, R. (1993). Une autre lecture de Nelligan. Voix et Images, 18(2), 378-381. https://doi.org/10.7202/201030ar d'utilisation que vous pouvez consulter en ligne.

https://apropos.erudit.org/fr/usagers/politique-dutilisation/ 
l'article *Postmodernisme" et "Vulgarité", qui parlent aussi de JeanFrançois Lyotard) porte justement le titre "Vulgarités". Voilà donc un moraliste qui se souvient de Parti pris et de cette chronique où les partipristes, dans les premiers numéros de la revue, se plaisaient à être bêtes et méchants. Guy Laflèche s'insère dans des lignées tout à fait honorables.

1. Guy Laflèche, Polémiques, Laval, Éditions du Singulier, 1992, 319 p.

2. Id., Les Saints Martyrs canadiens. Le Martyre de Jean de Brébeuf selon Paul Ragueneau, Laval, Éditions du Singulier, 1991, 342 p.

3. Voir aussi la note 33 , chapitre 11 , p. $235-239$, la note 19 , chapitre 12 , p. 263-265 et tout le début du *Glossaire - (p. 279 s.) sur cette question de l'économie missionnaire.

4. Contrairement à Guy Laflèche, je ne crois pas que le titre de la traduction française soit particulièrement : beau - (p. 63, note 30). On a traduit acivil disobedience - par - désobéissance civile *, ce qui est bien la moindre des choses, mais en oubliant le mot et la notion de -devoir, qui est au cœur du propos de Thoreau. L'homme juste, l'homme de principe, l'homme tout court (mais il n'y en a plus en Amérique, selon Thoreau) a le devoir de rompre avec son gouvernement quand les lois de l'État sont iniques. Le titre français cité par Guy Laflèche est tronqué et réducteur.

5. En criant à la censure. Mais cela n'est pas toujours convaincant. Quand un directeur de revue lui demande, afin d'en modifier le ton, de asoustraire ou de refaire. (p. 232) deux alinéas dans un article de vingt-sept pages, cela lui semble une ingérence intolérable. Dans ces conditions, combien de revues, combien de professeurs, combien de directeurs de thèse, qui font honnêtement leur travail de lecture et de révision en disant, qui à leurs collaborateurs, qui à leurs étudiants, de retravailler leur texte, sont de répugnants censeurs? Or, Guy Laflèche est le premier à affirmer: : Bien entendu, aucun mauvais livre ne doit être épargné et la critique doit toujours être absolument sans pitié. (p. 150). Ses articles seraient-ils les seuls à échapper au lot commun? Cette critique impitoyable doit aussi s'exercer sur un texte inédit, sous peine d'être un vœu pieux.

\section{Une autre lecture de Nelligan}

\section{Roger Chamberland, Université Laval}

La vie et l'œuvre d'Émile Nelligan ont été passées au crible de toutes les méthodes critiques en études littéraires: psychocritique, sociocritique, sémiotique, thématique, mythocritique, et j'en passe. Que nous reste-t-il à connaître d'une œuvre et d'un poète qui, visiblement, posent un problème d'analyse et d'interprétation à tout un pan de la critique qui cherche désespérément à faire coïncider drame existentiel et texte poétique. De fait, l'œuvre de Nelligan a connu un destin 
curieux: préparée par son auteur, elle a été revue et corrigée (censurée?) par son mentor, Louis Dantin, qui l'a publiée en 1904, et rééditée à plusieurs reprises, apportant chaque fois des corrections et des modifications. Ces éditions ont longtemps servi de référence jusqu'à ce que Luc Lacourcière publie le recueil des Poésies complètes, en 1952, qui ajoute 55 poèmes aux 107 déjà connus et remodèle la présentation afin de tenir compte des intentions du poète indiquées sur le manuscrit original en plus d'établir la date d'écriture ou de publication de la majorité des poèmes. Quarante ans plus tard, c'est au tour de Paul Wyczynski et Réjean Robidoux de présenter leur édition des Poésies complètes $^{1}$ qui vient enrichir et compléter l'édition Lacourcière. Ces éditions, sauf la dernière, seront d'une grande utilité pour Pierre $\mathrm{H}$. Lemieux dont l'étude, Nelligan amoureux ${ }^{2}$, propose "un nouvel itinéraire de lecture essentiellement centré sur les figures féminines. L'une des seules figures féminines sur laquelle la critique s'est longtemps fixée est sans aucun doute celle de la mère ${ }^{3}$, mais l'essai de Lemieux en suggère une autre vision et propose une interprétation bien différente qui prend appui sur l'analyse informatisée du vocabulaire de la poésie de Nelligan. Certes, ce croisement de données lui permet d'analyser les motifs récurrents et d'établir une liste privilégiée , de termes choisis. Il est dommage qu'il n'ait pas jugé bon de nous donner les paramètres de ce programme, ni même le tableau récapitulatif de ce travail. Mais cette lecture reste partielle si, comme le souligne l'auteur, on ne prend pas en considération "le sens littéral et obvie" des poèmes. Autrement dit, il entend privilégier l'analyse textuelle ou interne, "celle qui scrute minutieusement les textes pour aller $\mathrm{y}$ chercher les thèmes ou le poète, grâce à des recoupements thématiques, des similitudes de vocabulaire, des indications temporelles ou saisonnières, etc.v (p. 21). Si l'auteur affirme avec force vouloir s'en tenir aux textes, il n'écarte pas pour autant le recours à un "noyau historique dûment attesté par les biographes", principalement pour les chapitres 2 et 3 , respectivement consacrés à "La vierge blonde" et à "La vierge noire ". C'est dire que la biographie jouera, malgré tout, un rôle capital puisque c'est là que l'auteur ira chercher toutes les preuves pour appuyer sa lecture. La datation des poèmes est donc essentielle pour établir le parcours amoureux de Nelligan, car il y a coïncidence entre l'écriture des poèmes et des événements malheureux qui se sont passés dans sa vie.'

L'essai de Lemieux est divisé en trois chapitres correspondant chacun à une étape décisive dans la * carrière * amoureuse de Nelligan et à une période d'expression poétique particulière. La première, assurément la plus obscure, et celle de la "contestation" (octobre 1895-automne 1897), présente un Nelligan bohème, épris d'une 
"bergère", cette "Vierge blanche et rose", que Lemieux décrit comme * une fille pauvre et peu instruite, d'origine suisse-allemande, née dans un petit bameau pauvre mais fier, accroché au flanc du Mont-Royal* (p. 110), qui serait morte, laissant dans le désarroi le poète qui n'a jamais pu lui avouer ses sentiments. Cette étape est marquée par l'utilisation d'une mythologie littéraire païenne et l'emploi d'une imagerie bucolique jusque-là insoupçonnée dans l'œuvre de Nelligan.

La seconde étape est celle de "La vierge blonde" et de la "conversion" (automne 1897-printemps 1899). Le poète est amoureux fou de cette "Gretchen" qui ne lui rend pas son amour et l'abandonne à son triste sort d'amant éconduit (voir "Gretchen la pâle"). Nelligan opère donc un retour à la religion catholique, renonçant aux plaisirs de la chair et associant la femme à l'idée du mal et de la corruption ainsi que le prescrivait l'idéologie religieuse de l'époque. Lemieux appuie son argumentation sur le poème *Frère Alfus * où la critique a longtemps vu la représentation poétique du drame spirituel du meilleur ami du poète, Louis Dantin, alors qu'au contraire il s'agissait de sa propre crise de foi!

C'est à ce moment précis que se joue tout le drame de Nelligan qui renonce à l'amour et développe une "affreuse misogynie qui marque le début de la troisième période, celle dite de "La vierge noire" et de "l'aliénation. (juin-août 1899). Cette fois, l'amour de Nelligan se porte sur Françoise (pseudonyme de Robertine Barry) une amie de la famille - à quii il dédie et offre quelques poèmes, mais qui lui refuse toute forme de rapprochement. Dès lors, le poète manifeste une "méchanceté totale envers la femme et ne trouve aucun réconfort dans la religion: même la poésie ne peut lui servir d'exutoire et il devient ' 1 'horrible ronde des visions et des hallucinations" (p. 24). Ce qui permet à Lemieux de conclure que "Nelligan rejette alors le suicide et opte pour la folie ou l'aliénation définitive, mettant ainsi un terme à une courte mais foudroyante carrière poétique. (p. 24). Une telle affirmation ne manque pas de surprendre et laisse croire que Nelligan a délibérément choisi la folie, à défaut d'être chrétien et amoureux pourrait-on ajouter. D'ailleurs les affirmations de ce genre ne manquent pas dans cet essai et paraissent comme des raccourcis à une interprétation qui oscille entre l'analyse textuelle et les données biographiques. Le problème avec un tel va-etvient de l'œuvre au "noyau historique" est qu'il tombe sous le coup de la spéculation interprétative puisque nous n'avons aucun journal, aucun écrit théorique, aucun artefact convenable qui permette de dire avec certitude que tel événement s'est effectivement produit ou que le poète poursuivait tel ou tel projet en écrivant ses poèmes. De plus, le 
témoignage de contemporains est lui aussi sujet à caution, compte tenu du fait que Nelligan est en quelque sorte le "génie" qu'il faut protéger contre les dénigreurs, les dilettantes qui ne cherchent qu'à diminuer son importance afin de s'accaparer un peu plus de prestige, de capital symbolique dirait Bourdieu, grâce auquel il serait possible de prendre la place de ce poète maudit.

La lecture de Pierre H. Lemieux présente un portrait de Nelligan jusque-là insoupçonné. La vie amoureuse de ce dernier a donné lieu à plusieurs hypothèses: relation d'homosexualité avec cet autre bohème qu'était Arthur de Bussières, amour illicite avec sa mère, célibataire incapable de s'engager sentimentalement; bref, dans le domaine des passions du poète, la critique reste indécise. Pourtant, malgré tout le travail d'établissement d'une trajectoire amoureuse fait par Pierre $\mathrm{H}$. Lemieux, on ne peut adhérer à son hypothèse qui, bien que séduisante, repose sur une analyse qui fixe l'interprétation trop souvent sur les données biographiques. Ne s'agit-il pas là d'un réductionnisme à outrance? D'une matière d'aborder les œuvres que l'on croyait révolue? La poésie est ici réduite à un journal codé, où tout prend valeur de symboles, dans lequel le poète nous fait part des aléas et des avatars de sa vie amoureuse. Pourquoi tant insister sur la nécessité de s'en tenir à une analyse textuelle ou interne et sauter sur la première occasion pour aller chercher une "justification" du côté de la biographie? On veut bien croire que Nelligan ait connu une vie amoureuse commune aux adolescents de son âge, mais de là à faire reposer sa - folie. sur des déceptions amoureuses dont nul historien n'aurait relevé de traces me semble d'une grande témérité. Et si Nelligan s'était inspiré des tableaux d'époque, représentant des scènes bucoliques, qui ornaient peut-être la maison familiale, celle d'un ami, ou que l'on retrouvait dans certains lieux publics comme au Château de Ramezay? Et si Nelligan avait rêvé tout cela?

1. Il est pour le moins curieux qu'une édition critique visant à l'exhaustivité et se disant définitive gomme des articles majeurs - dont l'un signé de la main même d'un des auteurs - parus dans le Dictionnaire des ceuvres littéraires du Québec: l'un de Paul Wyczynski, (tome II), p. 407-41, l'autre d'André Gaulin, (tome III), p. 790-796. C'est lors de la recherche bibliographique pour la rédaction de l'article de Paul Wycynski, que nous avions découvert et reproduit un poème inédit de Nelligan, \&Vasque, ce que les auteurs de l'édition critique semblent avoir complètement oublié. Voir le compte rendu d'André Gervais panu ici même dans le numéro d'automne 1992

2. Pierre H. Lemieux, Nelligan amourewx, Montréal, Fides, 1991, 287 p.

3. Jacques Michon dans Emile Nelligan. Les racines du rêue Montréal, Les Presses de l'Université du Québec, 1983, arrive d'ailleurs à la conchusion que ala folie de Nelligan nous révèle que son rapport à la mère était plus profond que ses textes ne le laissaient soupçonner = (p. 83). 